Meta

Journal des traducteurs

Translators' Journal

\title{
Traduction et politique langagière au Japon : de l'" ouverture au monde " à la « mondialisation "
}

\section{Natalia Teplova}

Volume 51, numéro 4, décembre 2006

La traduction des noms propres (1) et Langue, traduction et mondialisation : interactions d'hier, interactions d'aujourd'hui Language, Translation and Globalization: Interactions from Yesterday, Interactions from Today (2)

URI : https://id.erudit.org/iderudit/014340ar

DOI : https://doi.org/10.7202/014340ar

Aller au sommaire du numéro

Éditeur(s)

Les Presses de l'Université de Montréal

ISSN

0026-0452 (imprimé)

1492-1421 (numérique)

Découvrir la revue

Citer cet article

Teplova, N. (2006). Traduction et politique langagière au Japon : de l'« ouverture au monde » à la " mondialisation ». Meta, 51(4), 758-770.

https://doi.org/10.7202/014340ar
Résumé de l'article

En cette époque dite de " mondialisation ", plusieurs pays voient leur(s) langue(s) nationale(s) menacée(s) par les langues de prestige. Cependant, le Japon présente un exemple de pays dont la langue-culture arrive à éloigner cette menace. Cet article propose d'observer, à travers l'histoire des réformes langagières, le développement de la langue japonaise écrite, ainsi que le rôle que joue la traduction dans ce processus. 


\title{
Traduction et politique langagière au Japon : de l'«ouverture au monde» à la «mondialisation »
}

\author{
NATALIA TEPLOVA \\ Université Concordia, Montréal, Canada \\ nteplova@alcor.concordia.ca
}

\begin{abstract}
RÉSUMÉ
En cette époque dite de «mondialisation», plusieurs pays voient leur(s) langue(s) nationale(s) menacée(s) par les langues de prestige. Cependant, le Japon présente un exemple de pays dont la langue-culture arrive à éloigner cette menace. Cet article propose d'observer, à travers l'histoire des réformes langagières, le développement de la langue japonaise écrite, ainsi que le rôle que joue la traduction dans ce processus.
\end{abstract}

\begin{abstract}
In this so-called age of "globalization," many countries see their national language(s) being threatened by more powerful rivals. Japan, however, is an example of a country whose language-culture manages to distance itself from that threat. This paper attempts an observation, through the history of language reforms, of the development of the Japanese written language, as well as of the role played by translation in that process.
\end{abstract}

\section{MOTS-CLÉS/KEYWORDS}

Japon, langue, réforme, histoire, mondialisation

\section{Introduction}

En cette époque dite de «mondialisation », l'impossibilité où se trouvent les États, les cultures, les langues même d'exister en vase clos semble être une évidence. Même la résolution de problèmes de politique intérieure (changement du gouvernement en Ukraine ou en Géorgie, entre autres), peu importe leur importance ou leur gravité, nécessite l'apport d'une opinion externe et, parfois même, elle oblige à la confrontation avec les valeurs de l'étranger. En ce qui a trait à la langue, la «mondialisation» a fini par être perçue comme une invasion des langues de prestige, qui menacent les langues nationales des pays touchés par le phénomène. Certaines langues-cultures parviennent cependant à éloigner cette menace. Le cas du Japon en est un bon exemple. L'histoire de ce pays, qui a pourtant connu plusieurs siècles d'isolement, est fortement marquée par sa relation avec l'étranger. Plus particulièrement, la langue japonaise écrite n'aurait jamais pu prendre sa forme actuelle sans l'influence de l'étranger, notamment des langues étrangères. Nous proposons d'observer, à travers l'histoire des réformes langagières, le rapport entre le japonais et les langues étrangères et le rôle que joue la traduction dans ces réformes.

\section{Formation de la langue japonaise écrite}

La langue japonaise moderne écrite comprend deux ensembles de symboles graphiques, différenciés par leur forme et par leur fonction. Le premier ensemble (kanji, 
qu'on peut traduire littéralement par «signes chinois») est emprunté à la langue chinoise et représente les morphèmes. Le second ensemble de symboles graphiques (kana) est un dérivé des caractères chinois, mis au point par les Japonais eux-mêmes. Kana est à son tour constitué de deux systèmes phonétiques syllabiques distincts, soit katakana et hiragana. Le premier est principalement utilisé pour la transcription des mots et des noms propres d'origine étrangère. Le fonctionnement du deuxième système phonétique est plus complexe. Hiragana sert surtout à la transcription des morphèmes qui ne sont pas exprimés par kanji, tels que les terminaisons des verbes conjugués ou des adjectifs déclinés. Ainsi, le texte en japonais moderne standard est toujours constitué d'une combinaison de kanji et de kana. Soulignons qu'à ces deux grands ensembles de symboles graphiques s'ajoute l'utilisation, quoique limitée, de l'alphabet romain (romaji).

\begin{tabular}{|l|l|l|}
\hline \multicolumn{2}{|l|}{ Système d'écriture japonais } \\
\hline $\begin{array}{l}\text { Caractères chinois } \\
\text { Kanji }\end{array}$ & $\begin{array}{l}\text { Syllabaires } \\
\text { Kana }\end{array}$ & Hiragana \\
\hline Kanji & Katakana & \\
\hline
\end{tabular}

La structure complexe du système d'écriture japonais ne s'est pas développée rapidement; au contraire, son histoire couvre près de deux mille ans. C'est en effet au $\mathrm{IV}^{\mathrm{e}}$ siècle (période Kofun, 250-600) que l'écriture chinoise pénètre au Japon par l'intermédiaire de la Corée, et l'art d'écrire est reconnu officiellement à partir du $\mathrm{V}^{\mathrm{e}}$ siècle (début de la dynastie Yamato). L'emprunt des caractères chinois pour créer un système d'écriture n'a rien de naturel ni de spontané; il s'agit plutôt d'une construction subjective voulue, bien pensée et bien contrôlée. De plus, il n'est aucunement question d'emprunter la totalité des signes chinois. Deux moyens, principalement, permettent de procéder à cette importation. D'une part, on accepte la signification de l'idéogramme choisi, sans trop prêter attention à sa prononciation chinoise. D'autre part, on choisit un caractère de manière plutôt arbitraire, uniquement parce que sa prononciation ressemble plus ou moins à celle du mot japonais existant, tout en rejetant la signification chinoise de l'idéogramme. Le premier mode d'emprunt contribue à enrichir le vocabulaire de la langue japonaise, tandis que le second vise à exprimer les réalités propres à la culture Yamato. Souvent, le même caractère finit par avoir une ou plusieurs prononciations chinoises (on-yomi) et japonaises (kunyomi).

Dès le début de l'élaboration de l'écriture, les Japonais savent que ce système est loin de l'idéal. Plus encore, ils réalisent combien la langue écrite et la langue parlée sont éloignées l'une de l'autre, voire dissemblables. Il n'est donc pas étonnant que le nouveau système se propage plutôt lentement parmi l'aristocratie japonaise et qu'il demeure mal défini pendant plusieurs siècles. Par contre, il peut paraître bizarre que la traduction n'ait eu aucun rôle à jouer dans le développement de l'écriture. Or, d'après Nitobe Inazo (1929: 9), les Japonais étant surtout impressionnés par l'économie du système chinois où un monosyllabe représenté par un seul caractère exprime une idée, ils ne sentent pas la nécessité de traduire. L'absence de la traduction est toutefois révélatrice sur les plans politique et idéologique. $\mathrm{Du} \mathrm{IV}^{\mathrm{e}}$ au vir siècle, le Japon n'est pas encore un pays unifié, mais les grands clans s'efforcent de protéger 
leur territoire contre les agresseurs externes et de consolider leur pouvoir. En évitant la traduction, on évite également l'émergence de dichotomies telles que «texte original/ traduction », "extériorité/intériorité», par exemple. Or, l'une des caractéristiques du mode d'importation de toute idée étrangère au Japon est justement l'effacement de l'origine de cette idée, au point où elle «devient» une réalité indigène. Grâce à ce procédé d'adaptation et à la prudence de l'aristocratie japonaise, qui évite les liens trop étroits avec les pays étrangers, le Japon réussit à éloigner la menace d'invasion idéologique étrangère et ce, malgré l'emprunt de l'écriture chinoise, du modèle chinois de bureaucratie et l'arrivée du bouddhisme.

Pourtant, si ni l'emprunt ni l'écriture des textes en caractères chinois ne suscitent le besoin de traduire, il en va tout autrement de la lecture. La grammaire japonaise et, par conséquent, la structure des phrases, est très différente de la grammaire de la langue chinoise. Même si les élites chinoise, japonaise et coréenne connaissent le kambun (écriture en chinois littéraire), ce qui facilite leur compréhension mutuelle, l'utilisation de cette écriture crée un écart profond entre la langue écrite et la langue japonaise parlée. D'ailleurs, il est tout simplement impossible de lire kambun en japonais en conservant, par exemple, la même structure ou en suivant l'ordre des mots. Afin de lire le texte chinois à la japonaise, on doit employer un autre système de signes de lecture (kaeri-ten). Ces signes, écrits à côté des kanji, indiquent l'ordre des mots pour la prononciation en japonais. À la lecture, on doit également ajouter les terminaisons grammaticales nécessaires qui ne figurent pas dans le texte original. Ainsi, en lisant le texte chinois, surtout à voix haute, on ne fait rien d'autre que le traduire.

Bien évidemment, les Japonais apprennent non seulement à lire, mais également à rédiger les textes en kambun. Cependant, le manque d'outils pour rendre compte de la grammaire, de la structure et de l'originalité de la langue japonaise se fait fortement sentir, surtout en poésie. À la fin du viıI ${ }^{\mathrm{e}}$ siècle, par exemple, on tente d'utiliser les caractères chinois de manière purement phonétique (mana) pour la composition de Manyōshū, première anthologie de la poésie japonaise. Puis, au $\mathrm{IX}^{\mathrm{e}}$ siècle, apparaît enfin le système d'écriture syllabique kana, qui vient remédier à la situation. Désormais, on ajoute par écrit les terminaisons grammaticales (okurigana) et on modifie l'ordre des mots. Cependant, les textes chinois demeurent non traduits et on conserve le style kambun, considéré comme plus officiel, pour la rédaction des textes bureaucratiques et des traités savants, entre autres. Kana est plutôt réservé aux compositions poétiques et aux textes de moindre importance. De plus, kambun est perçu comme un style d'écriture masculine et kana, comme un style plutôt féminin. Ces deux systèmes coexistent pendant plusieurs siècles, s'entremêlant souvent (kanjikanamajiri), mais il faut attendre le XVIII ${ }^{\mathrm{e}}$ siècle avant que soient traduits les textes écrits en kambun. C'est le personnage politique et philosophe Ogyū Sorai (1666-1728) qui, dans son ouvrage intitulé Yakubun Sentei (Aide à la traduction), rédigé en 1711, propose pour la première fois de traduire les textes écrits en kambun au lieu d'utiliser le système encombrant des signes de lecture.

\section{Langue et « ouverture» du pays}

La proposition d'Ogyū Sorai arrive au moment où le gouvernement du Japon, bien qu'il maintienne la politique d'isolement du pays (sakoku), ne peut plus faire semblant 
d'ignorer l'existence et l'influence de l'étranger. Comme nous l'avons déjà vu, le Japon entretient des liens très étroits avec la Chine et la Corée; ses relations avec l'Occident, quant à elles, remontent au $\mathrm{XvI}^{\mathrm{e}}$ siècle. C'est saint François-Xavier, missionnaire jésuite espagnol, qui, en 1549, a introduit non seulement le christianisme, mais également l'alphabet romain. En fait, il importe de préciser que le Japon n'arrive pas à s'isoler complètement. C'est surtout vers la fin de la période Tokugawa, quand l'intérêt envers la Hollande, par exemple, prend la forme de la science Rangaku («études hollandaises ») que l'impossibilité de l'isolement devient de plus en plus évidente. La traduction s'impose d'elle-même grâce à l'acceptation de l'existence de l' "Autre» et de l'extériorité. Cette même époque des Tokugawa voit apparaître les premiers traducteurs, qui sont des bureaucrates occupant des fonctions héréditaires au service du shogun. Cependant, puisque la traduction, comme le dit Sakai Naoki (1997), crée la différence à partir de l'incommensurabilité, une nouvelle nécessité apparaît: celle de définir le «Soi», l'intériorité. Ainsi recommence, avec une nouvelle force, le travail sur la langue japonaise. Par exemple, c'est la traduction et l'apparition des dictionnaires bilingues qui, en 1720, incitent le shogun Tokugawa Yoshimune à ordonner la compilation du dictionnaire de la langue nationale. Un peu plus tard, toujours pendant la période Tokugawa, naît le mouvement des études nationales (kokugaku) qui cherchent à définir les racines culturelles du pays et la tradition indigène japonaise en se basant non pas sur les textes confucianistes importés, comme le veut le shogunat, mais sur les textes anciens de la littérature japonaise. C'est ainsi que Motoori Norinaga (1730-1801), un des plus grands penseurs du XVIII ${ }^{\mathrm{e}}$ siècle et l'un des représentants du mouvement des études nationales, retraduit en japonais contemporain Kojiki (Le récit des événements anciens), un texte daté de 712.

Mais bien que les traductions intralinguistiques soient les plus réussies du $\mathrm{XVIII}^{\mathrm{e}}$ siècle, ce sont les traductions interlinguistiques qui relancent le débat sur le statut et le système de la langue japonaise. Ainsi, les traducteurs, bien placés pour mettre à jour et analyser les différences entre les langues et leur fonctionnement, sont parmi les premiers à proposer des réformes de la langue, principalement du japonais écrit. Maejima Hisoka (1835-1919), traducteur au Bureau du développement (Kaiseijo), organisme gouvernemental chargé des études occidentales, est le premier à suggérer l'abolition des caractères chinois. Dans sa pétition présentée en 1866 au shogun Tokugawa Yoshinobu, Maejima réclame le remplacement du système de kanji par celui de kana. La raison en est très pragmatique:

L'abolition de kanji (caractères chinois) dans l'éducation générale écourterait le temps perdu à mémoriser et à étudier l'écriture des caractères. [...] Sans aucun doute, il serait grandement avantageux que les [étudiants] consacrent le temps gagné à leurs études ou au travail, selon leur propre inclination. (Seeley 1991: 139)

Cette pétition, qui arrive au moment où le dernier shogun mène son ultime bataille pour conserver le pouvoir suprême, est rejetée, mais l'idée de Maejima laisse des traces profondes au sein des mouvements de réforme de la langue japonaise. Le shogun abdique l'année suivante et en 1868 commence la période Meiji (1868-1912) qui marque le début de l'histoire moderne du Japon.

Cette modernisation, cette «ouverture» du Japon (kaikoku) n'est rien d'autre qu'une occidentalisation du pays, qui se produit principalement par le biais de la traduction, puisque tous les réformateurs étant des traducteurs, spécialistes des études 
occidentales, le modèle occidental est le seul autre qu'ils puissent concevoir. Bien sûr, les nombreux traités signés avec les États-Unis, la Hollande, la Russie, la GrandeBretagne et la France assurent un contact direct avec ces pays et facilitent le choix du modèle à imiter. Il n'est donc pas surprenant que le slogan de l'époque soit Datsua $n y \bar{u} \bar{o}$ : «Loin de l'Asie, proche de l'Europe». Durant la période Meiji, l'initiative des réformes revient aux Japonais et pour moderniser le pays et rattraper les grandes puissances, les réformateurs proposent des changements, tant sur les plans politique et économique que langagier. Le mouvement d'unification de la langue écrite et de la langue parlée (Genbun'itchi) reprend l'idée de Maejima et recommande l'abolition des caractères chinois. Maejima lui-même entreprend en 1873 l'édition d'un journal quotidien en hiragana (Mainichi hiragana shimbun). En 1883, l'association savante Société de Kana (Kana no kai), qui publiera de nombreux ouvrages sur la question de réformes du système d'écriture, voit le jour. Il existe toutefois des solutions de rechange à l'adoption du système syllabique. Fukuzawa Yukichi (1834-1901), préoccupé surtout par la réforme du système de l'éducation, propose, quant à lui, de diminuer le nombre de caractères chinois. En 1873, pour illustrer son propos, Fukuzawa rédige un livre pour enfants en utilisant moins de mille kanji. Cependant, la proposition la plus radicale reste celle d'abolir l'écriture japonaise en faveur de l'alphabet romain. Formulée pour la première fois en 1869 par Nanbu Yoshikazu (1840-1917), cette idée est soutenue principalement par la société savante Meirokusha. Le tout premier article du numéro un de la revue Meiroku Zasshi, publiée par cette société, est consacré à cette question. C'est Nishi Amane (1827-1897), traducteur et grand penseur de la période Meiji, qui élabore la réforme radicale dans son article intitulé «Écrire le japonais en alphabet occidental»:

[...] ce que nous appelons les sciences et les arts apparaît seulement après l'existence des lettres. Sans lettres, comment peut-on avoir les sciences et les arts? [...] À présent, dans nos lettres, il nous est impossible d'écrire selon la manière dont on parle et de parler selon la manière dont on écrit. [...] Certains proposent qu'on diminue et détermine le nombre de caractères chinois (kanji). Les autres préferrent n'utiliser que le système syllabique japonais (kana). [...] je pense qu'il nous faut adopter l'alphabet [occidental] au complet. (Meiroku Zasshi 1976: 5-6)

Ironiquement, Nishi Amane, à qui on doit la création d'innombrables néologismes, n'utilise que les caractères chinois pour la conception du nouveau vocabulaire. Il traduit les concepts occidentaux à l'aide de mots composés (généralement de deux kanji), dissimulant en quelque sorte les origines étrangères des termes. Karatani Kōjin (1993: 18-23), un des intellectuels les plus marquants des vingt dernières années, appelle ce procédé «inversion»: les racines des mots sont brouillées et les réalités qu'ils représentent semblent être issues de l'espace de la langue-culture japonaise.

Le projet d'alphabétisation du japonais, repris et développé par Nishi Amane, a de nombreux partisans qui fondent, en 1885, l'association savante Romaji kai (Société d'alphabet romain). Fait intéressant, cette société compte parmi ses membres un étranger, Basil Hall Chamberlain (1850-1935), un des premiers spécialistes des études japonaises et traducteur de Kojiki, qui revendique l'emploi du style parlé en écriture. Nishi Amane établit une liste des dix avantages apportés par la réforme langagière, parmi lesquels le secours offert aux traducteurs et écrivains n'est pas le moindre. En effet, l'époque Meiji, surnommée «l'Âge des traductions», ne fournit pratiquement aucune méthode de traduction. Même les écrivains, ayant en quelque sorte perdu leur 
langue nationale, ne savent plus comment écrire. Ainsi, Futabatei Shimei (1864-1909), dont le roman Ukigumo (Nuages à la dérive) est considéré comme la première œuvre littéraire moderne japonaise, trouve son style à travers la traduction: après quelques années de travail sur son roman, Futabatei finit par rédiger la seconde partie de son œuvre en russe, puis la retraduit en japonais dans le style de la langue parlée ( $k \bar{g} g o)$. Une fois encore, comme à l'époque de l'emprunt à l'écriture chinoise, le japonais doit passer par une langue étrangère avant de se découvrir, de se définir.

\section{Réformes langagières $\mathrm{au} \mathrm{xx}^{\mathrm{e}}$ siècle}

Vers la fin du XIX ${ }^{\mathrm{e}}$ siècle, le mouvement Genbun'itchi, ainsi que la Société de Kana et la Société d'alphabet romain se trouvent dans une impasse, bien que leurs membres continuent de travailler aux nombreuses réformes qui transforment le pays. Il faudra attendre une autre "ouverture» du Japon (kaikoku), un autre grand contact avec l'Occident pour voir renaître le mouvement de réforme langagière, soit l'occupation américaine des années 1945 à 1952. Cette fois-ci, l'initiative n'appartient plus aux Japonais. C'est à la USEMJ (United States Education Mission to Japan) que revient, entre autres, la tâche de réformer le japonais. La grande question est: comment? Il n'y a pas là-dessus de consensus parmi les membres de la Division de l'Éducation, et c'est la voix de Robert King Hall qui se fait entendre. Hall cherche, entre autres choses, à «faciliter les échanges culturels par la simplification de la langue japonaise, qui rendra l'apprentissage de cette langue plus facile aux étrangers» (Wray 2001: 256). Hall réclame d'abord l'utilisation de katakana, puis finit par opter pour l'alphabet romain. Le ministère de l'Éducation du Japon (Monbushō) réagit et propose un compromis pour éviter les réformes extrémistes et l'organisme présente, en 1946, une liste uniformisée de 1850 kanji (Tōyō kanjihyō), ainsi qu'une liste de kana moderne (Gendai kanazukai). Mais, en raison de conflits internes au sein de la Mission américaine, les rêves de R. K. Hall ne se réalisent pas, et la langue japonaise réussit à conserver son système d'écriture. Quant à la standardisation des deux ensembles de symboles graphiques, elle était pour ainsi dire prête, grâce au Conseil délibératif en matière de la langue nationale, fondé par le ministère de l'Éducation en 1934; la campagne de Hall n'a fait qu'accélérer le processus. En 1948, les prononciations chinoise et japonaise sont déterminées pour chacun des kanji. En 1949, la forme des caractères chinois est définie et, en 1959, les règles d'emploi de okurigana (kana à emploi grammatical) sont adoptées. C'est entre 1946 et 1959 que se concrétise en grande partie la dernière réforme majeure de la langue japonaise.

Précisons toutefois que même si cette réforme est un succès, plusieurs révisions sont effectuées au cours des vingt années suivantes. En 1966, par exemple, le ministre de l'Éducation demande des précisions sur le choix des kanji pour la liste des caractères à usage quotidien (Tōyo kanjihyō), sur la standardisation des prononciations chinoise (on-yomi) et japonaise (kun-yomi), ainsi que sur l'emploi de okurigana. Après avoir produit en 1973 une nouvelle liste des kanji (Tōyō kanji onkunhyō) et une nouvelle méthode d'utilisation de okurigana (Okurigana no tsukekata), le Conseil délibératif en matière de langue nationale se rend compte que le travail de standardisation est loin d'être terminé. Il crée donc, un an plus tard, un nouveau comité chargé de l'étude et de l'élaboration d'une nouvelle liste de caractères. Il ne s'agit pas nécessairement d'augmenter le nombre des kanji, mais de travailler sur les détails qui permettront 
justement de mettre au point la standardisation des différents éléments du japonais écrit. En 1979, le comité met sa nouvelle liste à l'épreuve auprès du grand public et la soumet deux ans plus tard au ministère de l'Éducation sous le titre «Liste de caractères à usage courant » (Jōyō kanjihyō). La nouvelle liste de 1945 caractères est approuvée et son usage, promulgué le $1^{\text {er }}$ octobre 1981. Après environ cent ans de discussions et de recherches, les Japonais ont enfin une liste plus ou moins standardisée de caractères.

\section{Néologie et protection de la langue nationale}

On pourrait croire que, une fois la question des kanji résolue, la langue japonaise écrite a évacué pour de bon tous les problèmes liés à sa structure. Or, comme aucune langue vivante n'est stagnante, le japonais continue à subir des changements, à affronter les nouveaux problèmes imposés par l'époque et le contact avec les autres langues. Pendant les années 1980, un nouveau défi se pose donc, défi lié, cette fois, à la situation économique du pays. En effet, durant les deux dernières décennies du $\mathrm{xx}^{\mathrm{e}}$ siècle, le Japon vit un "miracle économique», qui le hisse au rang des grandes puissances mondiales. Or, vers la fin des années 1980, le pays, cédant aux pressions grandissantes des États-Unis, ouvre ses frontières et son marché intérieur aux produits et aux investissements étrangers. Le Japon entre alors dans une période d' "ouverture» et se pose en acteur important sur la scène de la mondialisation des marchés. Les problèmes de la langue japonaise sur le plan lexical réapparaissent donc en force, car en même temps que les produits et les capitaux étrangers, arrivent les emprunts linguistiques. $\mathrm{Si}$, pendant la période Meiji, la création de néologismes et l'introduction d'emprunts étaient surveillées par l'élite intellectuelle, tel n'est plus le cas à la fin du $\mathrm{xx}^{\mathrm{e}}$ siècle. Au contraire, le procédé est inversé: à cause des médias, de la culture populaire et d'Internet, les lexèmes étrangers s'intègrent à la langue-culture japonaise bien avant que les institutions ne remarquent ou ne contrôlent leur existence. Il revient aux organismes, comme le ministère de l'Éducation (Monbushō), l'Institut national de recherche sur la langue nationale (Kokuritsu kokugo kenkyūjo) ou le Comité des emprunts (Gairaigo iinkai) de répertorier les lexèmes qui inondent le pays, de les étudier et de rendre un verdict d'acceptation ou de rejet.

La plupart des emprunts viennent de l'anglais et, en cela, le Japon suit la tendance mondiale. Par ailleurs, cette situation reflète aussi l'histoire politique du Japon au $\mathrm{xx}^{\mathrm{e}}$ siècle. Vaincu lors de la Seconde Guerre mondiale, le pays n'a en effet eu d'autre choix que de se plier aux directives des États-Unis. Le slogan de l'époque de l'aprèsguerre est Datsua nyūbei: "Loin de l'Asie, proche de l'Amérique». Sous la pression de la Maison-Blanche, s'effectuent non seulement les réformes de la langue et de l'éducation, mais également l'élaboration de la Constitution. Toutes ces réformes et leurs conséquences entraînent des controverses, mais il n'en demeure pas moins qu'à la fin du $\mathrm{xx}^{\mathrm{e}}$ siècle, la tendance proaméricaine domine la politique extérieure du Japon. Cette situation n'est pas sans affecter la politique de la langue.

La nouvelle vague de gairaigo (littéralement «mots venus de l'extérieur») commence donc dans les années 1980 et marque le déplacement des préoccupations linguistiques: la problématique principale n'est plus du côté des signes chinois (kanji) et de leur standardisation, mais elle se situe plutôt dans l'ensemble du système phonétique syllabique (kana), et plus particulièrement dans le système de katakana. Comme nous l'avons déjà mentionné, katakana sert principalement à la transcription 
des mots et des noms propres d'origine étrangère. En 1954, le Conseil délibératif en matière de langue nationale décide de définir les règles orthographiques pour la transcription des emprunts en katakana et de contrôler le nombre de ces emprunts. Compte tenu du fait qu'en plus des noms propres d'origine étrangère, les onomatopées, certaines expressions familières et nombre de substantifs liés au domaine de la faune et de la flore sont écrits en katakana, il est facile de comprendre pourquoi le nombre des emprunts et leur présentation dans la langue japonaise écrite sont problématiques. «Traduire ou ne pas traduire?», telle est la grande question que se posent les institutions qui étudient le système d'emprunt. Lorsqu'on traduit un mot étranger, on finit le plus souvent par créer un néologisme composé de deux ou plusieurs kanji. Ce procédé a pour effet de cacher les racines étrangères du nouveau mot. Par contre, lorsqu'un emprunt est introduit en katakana, la provenance du mot reste clairement indiquée, ce qui n'empêche malheureusement pas sa signification de demeurer obscure. Le problème des emprunts en katakana présente ainsi deux facettes. D’une part, le nombre trop élevé de ces emprunts risque de bouleverser l'équilibre des ensembles de symboles graphiques de la langue japonaise écrite; d'autre part, la rapidité avec laquelle on introduit ces emprunts dans la langue pourrait dépasser le rythme auquel le grand public parvient à les assimiler.

Pour ces deux raisons, les institutions agissent avec précaution. En 2003, l'Institut national de recherche sur la langue nationale commence à publier dans le quotidien à grande diffusion Asahi Shimbun des listes de gairaigo, c'est-à-dire des néologismes transcrits en katakana, accompagnés de leurs traductions en kanji. De plus, les résultats de l'enquête sur la compréhension de ces lexèmes sont rendus publics. Avant de présenter la liste définitive des gairaigo acceptés et de leurs traductions, l'Institut tâte le terrain. Par exemple, le 25 avril 2003, après avoir interrogé 13000 personnes et organisé les mots en quatre niveaux selon le pourcentage de compréhension, l'Institut publie une liste de 62 emprunts acceptés. Puis, le 6 août 2003, il propose une nouvelle liste provisoire de 52 gairaigo qui doit elle aussi être mise à l'épreuve. À la suite d'une enquête, l'Institut publie, le 14 novembre 2003, la liste définitive de 47 lexèmes acceptés. Le rythme de la parution des listes est plutôt rapide, et, en observant le niveau de compréhension de la signification des emprunts en katakana, on remarque que le nombre de lexèmes compris par plus de $50 \%$ des répondants est limité: seulement 14 mots sur 62 figurant sur la première liste se trouvent dans cette catégorie. La troisième liste, la dernière en date, exige près d'un an de recherche avant d'être publiée. Une première version de 60 néologismes paraît dans Asahi Shimbun le 14 novembre 2003, mais la version définitive, comportant seulement 32 lexèmes, n'est affichée que le 8 octobre 2004 sur le site de l'Institut de recherche sur la langue nationale. Remarque intéressante: 31 de ces vocables sont compris par moins de $25 \%$ de la population.

À travers cette recherche, les Japonais n'expriment pas tant un rejet des gairaigo que leurs difficultés face à l'élément étranger. Ce phénomène est étudié par des spécialistes et analysé par les médias. Par exemple, le 18 février 2003, la chaîne de télévision nationale NHK diffuse une émission sur «Les mesures face à la croissance inattendue des mots en katakana» dans le cadre des reportages «Gros plan sur le temps présent ». Les spécialistes interviewés soulignent l'aspect historique des modes d'emprunt: en effet, on traduisait davantage pendant la période Meiji, tandis qu'aujourd'hui, on transcrit les mots phonétiquement de manière littérale. De plus, les spécialistes ont 
tendance à croire que la méconnaissance de la langue anglaise est à l'origine de l'incompréhension des emprunts.

Cette idée, par ailleurs, renvoie à un autre problème auquel fait face le Japon de la fin du $\mathrm{xx}^{\mathrm{e}}$ siècle, soit la réforme de l'éducation. En 1984, le premier ministre Nakasone exige une réforme de l'éducation afin de "répondre aux tendances de l'époque» où «l'internationalisation devient une question vitale» (Beauchamp 1994: 269). Le second rapport sur le développement de la réforme, publié en 1986, indique déjà que «l'enseignement de l'anglais au Japon est très inefficace» et que «l'enseignement de l'anglais en tant que langue nationale, non seulement en tant que langue internationale, ne doit pas être négligé» (Beauchamp 1994: 327). De toute évidence, la connaissance et la diffusion de la langue anglaise sont loin d'être perçues comme menaçantes; elles sont au contraire recommandées, encouragées et même exigées par les organismes gouvernementaux. Évidemment, certains s'y opposent. Il s'agit non seulement des linguistes puristes, mais aussi des nationalistes qui font valoir le statut unique de la culture et de la langue japonaises. (On ne s'étonne donc pas de voir apparaître plusieurs ouvrages sur la question, et leurs titres accrocheurs nous renseignent tout de suite sur la position de l'auteur. Par exemple, le titre de l'ouvrage de Tsuda Yukio, publié en 1996, n'exige aucune explication: Invasion de l'anglais, contreattaque du japonais: comment protéger la belle culture?). Mais, malgré les oppositions, la réforme de l'éducation et le mouvement de diffusion de l'anglais se poursuivent, et le gouvernement n'a élaboré aucune politique visant à limiter ou à interdire l'usage des emprunts à l'anglais. Il n'est pas impossible que cette situation se maintienne jusqu'au jour où, sait-on jamais, le nombre de gairaigo en katakana bouleversera l'équilibre du système d'écriture de la langue japonaise. Entretemps, la structure, la prononciation et le système d'écriture mêmes de la langue la protègent, puisque les emprunts sont confinés à un espace spécialement réservé aux éléments étrangers et ne peuvent, par conséquent, envahir l'autre ensemble de symboles graphiques.

Bien que les dictionnaires de néologismes en katakana soient toujours indispensables pour la compréhension de la terminologie contemporaine et que les emprunts assimilés en katakana ne soient pas acceptés trop rapidement, les lexèmes qui arrivent à passer dans l'usage participent à leur tour à la création de néologismes et à leur exportation dans l'espace anglophone. Le lexique lié à la culture populaire ou aux gadgets électroniques japonais n'est pas le seul présent dans les pays occidentaux; des concepts touchant à la nature même de la mondialisation le sont également. Par exemple, le terme anglais glocalization est en fait un lexème d'origine japonaise, introduit en 1983 par la compagnie Sony pour mieux décrire ses stratégies de marketing et d'expansion. Les gairaigo gurōbarizēshon (de l'anglais globalization) et rōkaru (de l'anglais local) sont déjà fortement ancrés dans la langue japonaise (bien que le premier n'apparaisse sur la liste officielle des emprunts acceptés qu'au mois de novembre 2003); ils peuvent ainsi servir à la création d'autres néologismes qui définissent plus clairement la volonté de l'entreprise de se servir de l'expertise locale lors de son expansion mondiale afin d'assurer son succès sur les marchés américain, asiatique et européen ciblés. D’ailleurs, l'emploi du mot glocalization, lexème accepté et assimilé en anglais, dépasse maintenant le domaine économique.

Le contact avec les langues-cultures étrangères à l'époque de la mondialisation pousse les intellectuels et les chercheurs de divers domaines à remettre en question des notions qui semblent pourtant bien définies et nommées, mais dont le sens change 
de manière radicale avec le temps. Au Japon, chaque nouveau contact avec l' «Autre» ramène à la redécouverte du «Soi». Ainsi, pendant des siècles, le mot désignant officiellement la langue japonaise est demeuré le lexème kokugo, qui se traduit littéralement par «langue nationale». Durant la longue période d'isolement du pays, ce mot ne posait pas de problème. Or, à cause de son ouverture au monde, avec la découverte et l'acceptation de l'«Autre», à la suite aussi de la participation du pays à l'internationalisation et à la mondialisation, le terme ne peut plus satisfaire aux exigences de l'époque. Le Japon reconnaît maintenant que chaque pays possède sa ou ses langue(s) nationale(s) et se voit obligé de choisir un autre lexème pour nommer la sienne. On pourra s'étonner que ce changement n'ait été effectué qu'en 2003. En effet, le 27 avril 2003, le journal Asahi Shimbun annonce la nouvelle sous la rubrique «Intersection des mots", dans un article intitulé " "Langue nationale" et "langue japonaise": la Société savante prend conscience des étrangers et change de nom». En effet, la Société savante de la langue nationale (Kokugo gakkai) décide de changer son nom pour «Société savante de la langue japonaise» (Nihongo gakkai) afin de mieux définir l'objet de ses études. Cependant, le même article nous apprend que les organismes gouvernementaux travaillant à des recherches semblables n'ont pas l'intention de prendre un autre nom. Force est de constater, une fois de plus, que les institutions bureaucratiques n'arrivent pas à suivre le rythme effréné des changements imposés par la mondialisation.

\section{Mondialisation et traduction}

Le Japon d'aujourd'hui ne se sent donc pas menacé par l'Occident ou, plus particulièrement, par les États-Unis sur le plan de sa politique langagière. La mondialisation est perçue comme une étape du processus d'américanisation et ce sont, entre autres, les thèmes de la démocratie, du droit des peuples à disposer d'eux-mêmes et de la liberté et de la sécurité personnelles qui constituent des sujets de discussion non seulement dans les milieux politiques et savants, mais également parmi le grand public. Par exemple, le quotidien Mainichi Shimbun commence à publier en janvier 2002 une série d'articles intitulée «Mondialisation: ombre et lumière». Cette série se compose de plusieurs parties, dont chacune présente à son tour divers articles réunis sous un même thème et rédigés par des spécialistes, japonais aussi bien qu'étrangers, de différents domaines. Ainsi, les quatre séries d'articles parues en 2002-2003 s'intitulent respectivement «Voie de la nation», "Société des corporations en changement», "Pour un siècle de l'humanité» et «Mode de vie "Amérique"». Peu importe le sujet ou l'origine de son auteur, chaque article a pour leitmotiv l'idée de respect, et la question de la langue revient régulièrement. Par exemple, ce sont les différentes facettes du problème de la langue qui sont abordées dans l'article de l'écrivain Kuroi Senji, paru le 30 septembre 2002 sous le titre «Dynamisme du globe». L'auteur analyse d'abord les termes global et globalization tels qu'ils se présentent dans la langue japonaise. En observant, dit-il, ces emprunts en katakana à l'ouïe sans en connaître le sens, il n'en ressort que quelques sonorités. Par exemple, les mots global et globalization «sonnent» comme le lexème japonais harubaru qui fait référence à une "grande distance à parcourir ». Or, si la transcription katakana ne véhicule aucun sens, ce n'est pas le cas des idéogrammes. Kuroi propose donc sa traduction littérale du terme globalization en japonais, le mot zenkyūka, composé de trois kanji: zen («totalité»), 
$k y \bar{u}$ («globe»), $k a$ («transformation»). La traduction de Kuroi se distingue légèrement de la version officielle de l'Institut national de recherche sur la langue nationale, qui propose plutôt le mot chikyūkiboka, composé de cinq kanji représentant trois syntagmes: chikyū ("globe»), kibo («échelle») et ka («transformation»). Mais peu importe la version préconisée, puisque chaque caractère étant porteur de sens, non seulement la notion est-elle clairement définie, mais on en sent aussi toute la profondeur. Une autre question, cependant, s'impose immédiatement: les Japonais aurontils la volonté de participer à ce processus de changement à l'échelle mondiale? Selon Kuroi, avant même de prétendre obtenir un résultat positif, tous les Japonais doivent prendre conscience de la situation mondiale et de l'économie des marchés. On doit aussi, souligne-t-il, être conscient du fait que le développement des nouvelles technologies repose sur l'échange énergique de l'information. Ainsi, selon Kuroi, les conditions nécessaires de mise en marche du processus de mondialisation sont l'action concertée et le maintien d'un haut niveau de communication et de dialogue. Bien qu'idéaliste, cette pensée, qui met en valeur le dialogue entre les pays, nous ramène à la question de la langue: l'auteur se demande en effet si la domination de l'anglais favorise la mondialisation ou si elle en est plutôt le résultat. Si l'anglais avait été la langue unique des êtres humains, aurait-elle eu une influence positive sur le processus de mondialisation? Si une langue commune s'était avérée nécessaire, n'aurait-il pas été plus efficace de privilégier une langue construite comme l'espéranto? Pour Kuroi, la multitude des langues et leurs différences sont des éléments inévitables de la mondialisation, et au cœur des mots global et globalization se cache une relation de tension profonde entre «altérité» et «homogénéité»; or, c'est justement cette dichotomie qui a le pouvoir de construire un «globe».

Si nous poussions cette réflexion un peu plus loin, nous pourrions suggérer que c'est principalement à travers la traduction que cette tension est ressentie le plus vivement. D'ailleurs, la traduction présuppose ici non pas un contact abstrait et purement linguistique, mais un contact réel des langues-cultures. À eux seuls, les différents modes d'emprunts lexicaux du japonais illustrent clairement qu'un gigantesque bagage culturel est transmis à ce contact, en plus de l'élément linguistique. Il n'est donc pas surprenant, que vers la fin du $\mathrm{xx}^{\mathrm{e}}$ siècle, on ait commencé à parler au Japon de «traduction de cultures» (bunka no hon'yaku); c'est en effet à travers la traduction que s'ouvre l'univers d'une langue-culture auparavant méconnue (Nakashima 2002: 7). Chaque nouvelle découverte constitue ainsi une pièce supplémentaire qui servira à la reconstruction du «globe».

\section{Conclusion}

Comme nous l'avons vu, chaque réforme langagière au Japon correspond à une série de contacts déterminants avec l'étranger, pendant lesquels la traduction (et parfois la non-traduction) joue un rôle important. La langue japonaise a chaque fois recours aux langues étrangères pour se renouveler et si, aujourd'hui, le système d'écriture ne semble plus menacé, le recours systématique aux langues étrangères est source de débats constants chez les intellectuels japonais. Cependant, la clé de la protection de la langue japonaise se trouve dans sa structure et son fonctionnement mêmes, dans sa capacité d'assimiler l'élément étranger soit en le confinant à l'espace rigide de katakana, soit en oblitérant ses origines, sitôt le mot japonais créé. 


\section{RÉFÉRENCES}

ANDERER, P. (ed.) (1995) : Literature of the Lost Home. Kobayashi Hideo - Literary Criticism, 19241939, Stanford, Stanford University Press.

Asahi Shimbun, 18 février 2003.

Asahi Shimbun, 25 avril 2003.

Asahi Shimbun, 27 avril 2003.

Asahi Shimbun, 6 août 2003.

Asahi Shimbun, 14 novembre 2003.

Beauchamp, E. R. and J. M. Vardaman Jr. (eds) (1994): Japanese Education since 1945. A Documentary Study, Armonk, M. E. Sharpe.

Block, D. and D. CAmeron (eds) (2002): Globalization and Language Teaching, London and New York, Routledge.

Brody, B. (2002): Opening the Door. Immigration, Ethnicity, and Globalization in Japan, New York and London, Routledge.

Bowman, J. S. (ed.) (2000): Columbia Chronologies of Asian History and Culture, New York, Columbia University Press.

Clammer, J. (2001): Japan and Its Others, Melbourne, Trans Pacific Press.

Hyun, T. and J. Lambert (eds) (1995): Translation and Modernization. Proceedings of the XIIIth Congress of the International Comparative Literature Association, Tokyo, ICLA.

IснікаWA, S. (1929): Foreign Influences in the Japanese Language, Tokyo, The Japanese Council, Institute of Pacific Relations.

IIDA, Y. (2002): Rethinking Identity in Modern Japan. Nationalism as Aesthetics, London and New York, Routledge.

Inoue, N. (ed.) (1997): Globalization and Indigenous Culture, Tokyo, Institute for Japanese Culture and Classics, Kokugakuin University.

Ітон, M. (1998): Globalization of Japan. Japanese Sakoku Mentality and U. S. Efforts to Open Japan, New York, Macmillan.

Iwabuchi, K. (2002): Recentering Globalization. Popular Culture and Japanese Transnationalism, Durham and London, Duke University Press.

Karatani, K. (1993): Origins of Modern Japanese Literature, Durham and London, Duke University Press.

KOKURITSU KOKUGO KENKYŪJO / THE NATIONAL INSTITUTE FOR JAPANESE LANGUAGE, (page consultée le 8 octobre 2004), <http://www.kokken.go.jp>.

КuвотA, R. (2002): “The impact of globalization on language teaching” in David Block et Deborah Cameron (ed.). Globalization and Language Teaching, London and New York, Routledge, p. 13-28.

Lincicome, M. E. (1995): Principle, Praxis, and the Politics of Educational Reform in Meiji Japan, Honolulu, University of Hawaii Press.

Loveday, L. J. (1996): Language Contact in Japan. A Socio-Linguistic History, Oxford, Clarendon Press.

Lovins, J. B. (1975): Loanwords and the Phonological Structure of Japanese, Bloomington, Indiana University Linguistics Club.

Mainichi Shimbun, 30 septembre 2002.

Meiroku Zasshi. Journal of Japanese Enlightenment, translated by William R. Braisted, Cambridge, Harvard University Press, 1976.

Miller, R. A. (1980): Origins of the Japanese Language, Seattle and London, University of Washington Press.

Miyanaga, K. (2000): Gurōbaruka to aidentiti (Mondialisation et identité), Tokyo, Sekaishisosha. Nagashima, Y. (2002): «'Bunka no hon’yakusha’ Mori Ōgai wo minaosu» («Traducteur de cultures: nouveau regard sur Mori Ōgai»), Mainichi Shimbun, 6 septembre, p. 7.

Nakayama, S. (1984): Academic and Scientific Traditions in China, Japan and the West, Tokyo, University of Tokyo Press. 
Nitobe, I. (1929): Foreign Langages in Japan. Their Use and Study, The Osaka Mainichi and The Tokyo Nichi Nichi.

Outline of Historical Materials Concerning Education Reform Under the Allied Occupation of Japan. A Joint Research Survey and Collection of Materials Mainly Through NIER Group, Tokyo, National Institute for Educational Research, March 1989.

Oshima, K. (2002): Gairaigo Usage in Japan: From Cultural Controversy to a New Analytical Framework, Tokyo, International Christian University.

SakaI, N. (1992): Voices of the Past. The Status of Language in Eighteenth-Century Japanese Discourse, Ithaca-London, Cornell University Press.

SAKaI, N. (1997): Translation and Subjectivity. On "Japan" and Cultural Nationalism, MinneapolisLondon, University of Minnesota Press.

Sato Habein, Y. (1984): The History of the Japanese Written Language, Tokyo, University of Tokyo Press.

Seeley, C. (1991): A History of Writing in Japan, Leiden, E. J. Brill.

Starrs, R. (ed.) (2001): Asian Nationalism in an Age of Globalization, England, Japan Library.

Starrs, R. (ed.) (2002): Nations Under Siege. Globalization and Nationalism in Asia, New York, Palgrave.

Tsuchimochi, G. H. (1993): Education Reform in Postwar Japan. The 1946 U. S. Education Mission, Tokyo, University of Tokyo Press.

Tsuda, Y. (1996): Shinryaku suru eigo hangeki suru nihongo: utsukushii bunka o dō mamoru ka (Invasion de l'anglais, contre-attaque du japonais: comment protéger la belle culture?), Tokyo, Pieichipi kenkyujo.

Tsurumi, S. (1986): An Intellectual History of Wartime Japan 1931-1945, London, New York, Sydney and Henley, KPI.

Unger, M. J. (1996): Literacy and Script Reform in Occupation Japan. Reading between the Lines, New York, Oxford, Oxford University Press.

Wray, H. (2001): "Nationalism, Cultural Imperialism, and the Language Reform in Occupied Japan" in StARrs, Roy (ed.). Asian Nationalism in an Age of Globalization, Richmond, Japan Library, p. 253-290. 